\title{
Airway Microbiota diversity and composition correlates with severity of Chronic Obstructive Pulmonary Disease (COPD)
}

Sara Dias, Filipa Machado, Carla Valente, Lilia Andrade, Alda Marques, Ana Sousa

ERJ Open Research 2019 5: PP224; DOI: 10.1183/23120541.lungscienceconference-2019.PP224

\section{Article}

Figures \& Data

Info \& Metrics

\section{Abstract}

COPD occurrence is mediated by immune response, environmental factors and microbial dysbiosis. Airway microbiota has been found to be different between healthy people and patients with COPD and these differences could influence disease severity. But, its clinical implications are still unclear. Here, we have explored the airway microbiota of patients with COPD as well as its relations with specific clinical parameters.

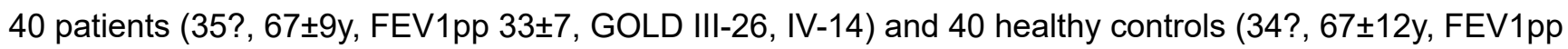

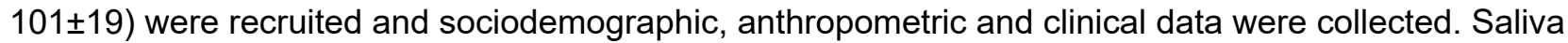
microbiota was characterised by $16 \mathrm{~S}$ rRNA sequencing and analysed using Qiime2 pipeline.

Significant differences in airway microbiota composition between patients and healthy people were observed (Fig 1). The microbiota of patients with worse clinical parameters, like lower FEV1pp and SpO2, or with higher number of exacerbations and hospitalisations, clustered separately from less severe. Loss of microbiota diversity correlated with age in patients, but not in healthy people, raising the hypothesis of the cumulative use of antibiotics (by patients) contributing to dysbiosis.

\section{WE USE COOKIES ON THIS SITE TO ENHANCE YOUR USER EXPERIENCE}

By clicking any link on this page you are giving your consent for us to set cookies. 


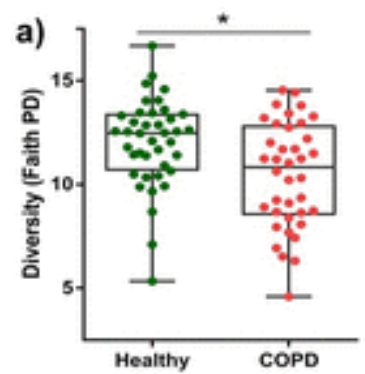

c)
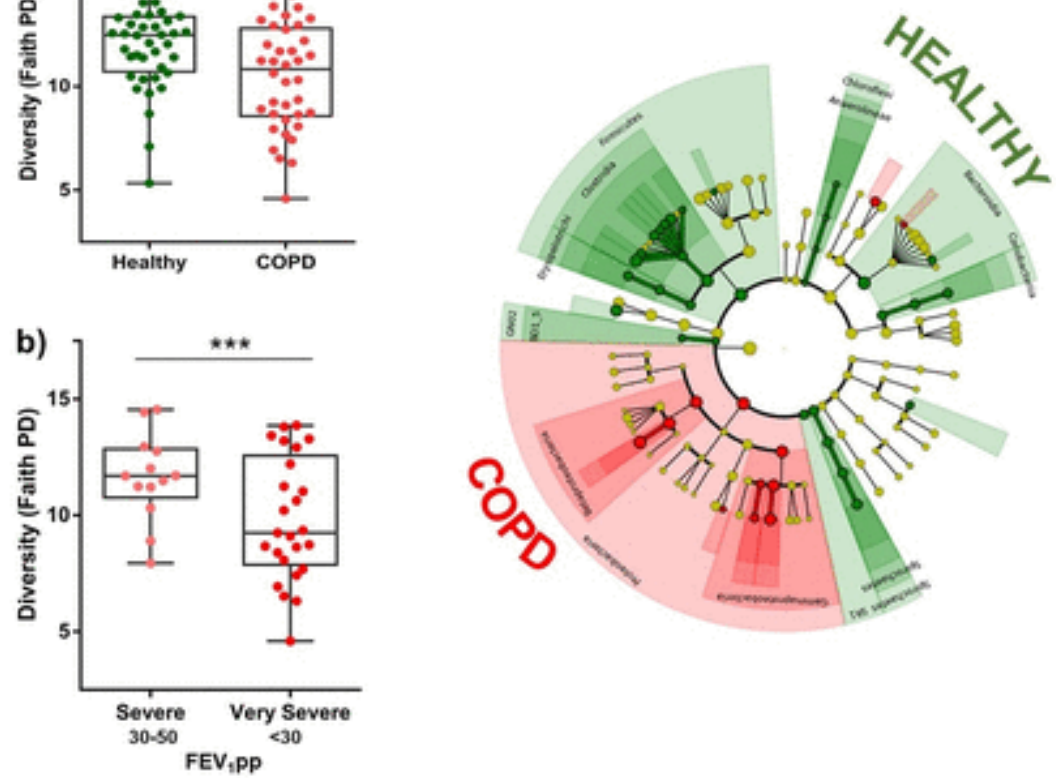

Fig 1 - Diversity and composition of the Airway microbiota and composition is different between healthy and COPD groups. a) The microbiota diversity (estimated as Faith's phylogenetic diversity $(P O)$ ) is significantly higher $(0<0.05)$ in healthy subiests than in patients, b) Estimated alpha diveryity, for COPO group according to ainway obstruction grade (FEV, pp). The microbiota from patients with very severe states of airway obstruction is significanaly less diverse (pe0.001) than in patients with less severe airway obstruction. c) The cladogram represents the comparison of COPD and Healthy group's microbiotas. At phylum level, patients' microbicta is enriched in Proteobocterio.

Download figure

Open in new tab

Download powerpoint

The association between microbiota composition and clinical parameters supports its contribution to COPD trajectory. Future studies should focus on remodelling patients' dysbiotic microbiotas towards healthier ensembles aiming at preventing disease decline.

\section{Footnotes}

Cite this article as: ERJ Open Research 2019; 5 : Suppl. 2, PP224.

This is an ERS Lung Science Conference abstract. No full-text version is available. Further material to accompany this conference is available at www.ers-education.org (ERS member access only).

Copyright @the authors 2019

\section{WE USE COOKIES ON THIS SITE TO ENHANCE YOUR USER EXPERIENCE}

By clicking any link on this page you are giving your consent for us to set cookies.

\section{OK, I agree}

No, give me more info 